\title{
Jóvenes universitarios y pre-universitarios y actitudes cívico-políticas hacia una Europa social
}

\author{
Carmen Viejo Almanzor ${ }^{*}$ ), Mercedes Gómez-López $\left(^{*}\right)$ y Rosario Ortega-Ruiz ${ }^{*}$ ) \\ (*) Universidad de Córdoba - España
}

\begin{abstract}
RESUMEN
La Universidad se ocupa de dotar a las nuevas generaciones de la capacidad para comprender ideas y procesos complejos, pero también de formar la personalidad de los y las jóvenes para ser capaces de identificar los retos para avanzar hacia un futuro mejor. Con el objetivo de describir el análisis crítico que la juventud española hace de la Europa actual y futura, y determinar el valor que otorga a la educación, se analizaron las prioridades y preocupaciones con respecto al futuro, las visiones sobre el futuro de Europa, los valores y las actitudes de 268 jóvenes de entre 16 y 25 años (64.6\% chicas, 35.1\% chicos, 0.4\% otro), con una edad media de 20.72 años. Los resultados señalaron el desempleo como una de las principales inquietudes, sólo superada por el terrorismo. Sin embargo, su visión sobre la Europa del futuro está marcada por la atribución de valor a la Educación, junto a valores democráticos, de atención y derechos a la diversidad, equidad y la justicia. Se discuten estos resultados con relación al valor que la Educación Superior tiene para la formación de un pensamiento crítico y la construcción de la Europa social que algunos de ellos deberán liderar.
\end{abstract}

Palabras Clave: Juventud, Universidad, Educación, Europa.

\section{University and pre-university students and civic-politics attitudes toward a social Europe}

\section{ABSTRACT}

The University is the institution responsible for providing new generations with the skills to understand and analyze complex ideas and processes. But it must also shape the personality of young people to be able to identify the potentialities of different realities and visualize the possible challenges to move towards a better future. This study aims to describe the critical analysis that Spanish youth make of current and future Europe, and to determine the value that they give to Higher Education as a tool for improvement and advancement of society. This study analyses the priorities, visions and worries about future, and the values and attitudes of 268 young people; they were aged between 16 and 25 years old (64.6\% females, 35.1\% males, $0.4 \%$ other denomination). The results indicated unemployment as one of the main concerns, only overpassed by terrorism. However, the expectations and motivations profile of Spanish youth regarding their future Europe is positive. Their vision is marked by the attribution of value to Education, together with democratic values, attention and rights to diversity, equity and justice. These results are discussed in relation to the value that Higher Education has for the formation of critical thinking and the construction of the social Europe that some of those youngers should lead.

Keywords: Youth, University, Higher Education, Europe.

La Universidad es una de las instituciones más antiguas y que más prestigio social tiene. Este crédito es debido, en gran medida, al reconocimiento de que, junto a la tarea de transmisión del conocimiento, tiene la de creación del propio conocimiento. En este sentido, el Informe Bricall (2000) destacaba la Universidad como institución social a la que se le atribuían un amplia variedad de funciones que podían resumirse en tres: la función socializadora, dedicada a la preservación y transmisión crítica del conocimiento, cultura y valores sociales; la función orientadora, dedicada al desarrollo de las capacidades de cada individuo; y la función investigadora y de extensión cultural, con la que se pretende el aumento de la base del conocimiento de la sociedad. Crear conocimiento pasa, por tanto, por dotar a las nuevas generaciones de la capacidad y las actitudes para comprender y analizar ideas y procesos complejos, pero también por formar la personalidad de los universitarios para ser capaces de identificar las potencialidades de realidades de distinta naturaleza y visualizar los retos e iniciativas posibles para avanzar hacia un futuro mejor. Se trata de formar profesionales competentes en un mundo cambiante, capaces de transformar el conocimiento especializado adquirido en la Universidad en herramientas para elaborar respuestas a las necesidades sociales (Brand-Gruwel, y 
Stadtler, 2011; Palmer, Montaño, y Palou, 2009). En este sentido, hay que entender que Bricall (2000) está incluyendo en la función orientadora mucho más que la simple guía del estudiante. Los estudios universitarios, tal y como históricamente se han configurado, tienen en la vieja titulación de licenciatura, hoy conocida como Grado, un imperativo orientativo que puede llegar a suponer un importante impacto en la personalidad del o la joven. No cabe duda de que los años universitarios, colocados de media en unas edades de juventud en la que los individuos son tan receptivos a la información, el conocimiento y las nuevas ideas que la universidad puede proporcionarles, impactarán en su formación y en la configuración de su personalidad en todos los sentidos, desde su competencia para abordar planes profesionales, hasta sus valores, su sentido de la vida, su ubicación como miembros de una determinada comunidad y sus proyectos vitales. A este respecto, es necesario destacar que, paralelamente a los y las jóvenes que ya forman parte del sistema universitario, también es labor de la universidad definir un espacio cultural que facilite la integración de nuevos/as alumnos/as (Ferra, López y Galiano, 2018). Factores como la adaptación a los nuevos procesos didáctico-curriculares, la autonomía y seguridad personal, o la integración académico-social del estudiante parecen ser clave para el buen desempeño en los años posteriores (Ferra, López y Galiano, 2018). En este sentido, la implementación del Espacio Europeo de Educación Superior (EEES) ha supuesto una transformación profunda del modelo formativo previo (Rodríguez-Izquierdo, 2014), promoviendo el papel activo del alumnado en su propio proceso educativo (López-Sidro, 2011). Estos cambios pueden suponer un mayor desafío para la adaptación exitosa de los y las jóvenes que acceden a un nuevo sistema, en el que deben aprender a desenvolverse y que supone un salto cualitativo de vital importancia (Rodríguez-Muñiz y Díaz, 2015). Por otra parte, también es importante considerar que la tecnología de la información y la comunicación está modificando el marco universitario en todas sus funciones. Dada la rapidez de transformación tecnológica y social de los últimos tiempos, las universidades europeas han tenido que afrontar un nuevo reto: dibujar un marco común de trabajo que diera respuesta a las necesidades reales de los y las ciudadanas del siglo XXI. No obstante, ha supuesto, a su vez, una oportunidad única para llevar a cabo los cambios necesarios para adaptar el sistema universitario a las verdaderas necesidades formativas que exige una sociedad moderna en un mundo global y altamente tecnológico (Santiago, Tremblay, Basri, y Arnal, 2008); una sociedad que exige a sus ciudadanos autonomía, responsabilidad personal y social, emprendimiento, liderazgo y solidaridad (Huber, 2008; Tobón, Rial, Carretero, y García, 2006). En este sentido, la educación universitaria ha de asumir un rol protagonista en cuanto se refiere a la formación de los y las jóvenes universitarios de hoy, que serán los y las ciudadanas de la Europa de mañana. La función formativa de la universidad en sus niveles básicos (el Grado) no puede obviar aspectos del rol protagonista que le atribuimos en la conformación de personalidades sociales, siempre diversas y libres, que al mismo tiempo tienen que registrar, entre sus valores, el de la universalidad del conocimiento, el respeto a los rasgos culturales del entorno en el que se mueven y, muy especialmente, valores de atención a la diversidad, los derechos humanos y la consideración igualitaria de todos los miembros de las diversas identidades culturales que componen las naciones y también las entidades supranacionales, como es el caso de la Europa actual.

Sobre esta premisa, la investigación en ciencia social está reconociendo la necesidad de estimular políticas y prácticas que den cobertura a necesidades e intereses humanos que vayan más allá de la corta visión nacional, asumiendo una mirada del mun- do como un todo globalizado (Jover, 2001; Kennedy, 1997). Un mundo globalizado que de momento ha mundializado la economía, el sistema capitalista y ciertos valores del mercado que se suelen tomar, acríticamente, por la población poco instruida. Evidentemente, las universidades del mundo que consideramos civilizado deben huir de estos elementos acríticos, bastante generalizados. Una mirada cosmopolita (Ortega, 2015) exige una comprensión de la globalización y la tecnologización del conocimiento que asuma que lo que hay que mundializar, más allá del comercio y las finanzas, son los valores universales de equidad y justicia. Esta mirada amplia y cosmopolita requiere un discurso que integre tanto lo local, en términos de las necesidades directas de los individuos, cualquiera que sea el lugar donde han nacido o donde desarrollan su vida, como lo global, en términos de una homogeneización tecnológica que acerque la competencia para comprender el mundo en su complejidad. El avance científico y tecnológico que se está produciendo desde finales del siglo XX no tiene precedentes, y el desarrollo de las tecnologías de la información y la comunicación es uno de los factores que mayor impacto está teniendo en el fenómeno globalizador, con un efecto positivo -estimula exponencialmente el desarrollo en cuanto que disemina la información-, y otro negativo -hace más competentes y sabios a los que acceden a una tecnología vanguardista, más y más sofisticada-. Actualmente es posible establecer comunicaciones impensables en otros momentos históricos (Marín, 2003). Europa es uno de los territorios donde estas tecnologías han penetrado con gran intensidad, lo que da lugar a modelos de comunicación e intercambio cultural que demandan una comprensión satisfactoria porque la comunicación y la información es uno de los factores más importantes del desarrollo. Europa es un crisol de culturas de profunda base histórica, complejas en sí mismas y cuyo entramado da lugar a un hermoso pero enigmático arcoíris cultual. Europa además tiene, en las instituciones supranacionales o comunitarias, la oportunidad de avanzar hacia más positivos modelos de internacionalismo, universalismo y progreso en el ámbito social y cultural. Analizar la complejidad del proyecto europeo exige comprender la profundidad de las culturas de los pueblos europeos al tiempo que se asume la universalidad del fenómeno de la globalización y el impacto que las tecnologías están proporcionando en la vida económica, social y política del proyecto europeo.

El papel activo que España desempeña en la construcción de un proyecto europeo común, a través de la contribución al desarrollo de políticas de cohesión social o en ámbitos como la diversidad cultural o la lucha contra el terrorismo, manifiesta su compromiso con el fomento de la identidad europea. Al considerar a la Unión Europea como el marco natural de desarrollo político y económico del país (MAEC, 2018), gran parte de la población española percibe a Europa y al proyecto de la Unión Europea como un gran reto, cuajado de dificultades, pero aun esperanzador y optimista. La educación es clave para el futuro de Europa, porque serán los jóvenes actuales los adultos que asumirán la compleja tarea de articular identidades hacia un proyecto común. Serán ellos, quienes están en pleno proceso de desarrollo y construcción de su propia identidad, en gran medida constituida desde sus orígenes más íntimos y culturales basados en las vivencias locales, los que, convertidos en jóvenes europeos legítimamente protagonistas de la tarea de construcción de la identidad europea, dibujen un futuro propio y al mismo tiempo global y cosmopolita para Europa. Cualquier identidad, ya sea individual o colectiva, requiere hacer una referencia a un colectivo mayor con el que se comparten determinados rasgos (Larrain, 2003). En un mundo cada vez más interconectado, los jóvenes amplían y desafían fronteras, construyen y deconstruyen nuevas 
y no tan nuevas definiciones sobre su condición vital y entremezclan valores, creencias, actitudes y formas de ser y actuar al desenvolverse en un contexto cada vez más multicultural.

No obstante, la juventud como colectivo social es una creación epistemológica reciente (Britto, 2004), un concepto cuyo significado se construye en función de las situaciones económicas, culturales y socio-políticas del momento histórico (Moreno, 2015). Diferentes posiciones teóricas convergen en entender la juventud como una etapa de tránsito en la que se adquieren las competencias y habilidades necesarias para incorporarse a la sociedad en un rol de adulto, y en la que el papel de la formación y la educación superior juegan una posición preponderante. Las variables que modulan esta incorporación varían en función del contexto en el que el/la joven se desenvuelvan: se establece una relación entre identidad y cultura, la cimentación de identidad es un proceso simultáneo material, social y cultural (Kaluf, 2005).

Desde esta mirada, se hace evidente que la crisis económica mundial también haya incidido de forma particular en la visión de los más jóvenes, dejando al descubierto fallos estructurales que conllevan graves consecuencias, como la elevada tasa de desempleo juvenil. Si bien en todos los países de la UE las tasas de desempleo de los jóvenes son, por lo general, más altas que en el conjunto de la población, en algunos estados miembros, supera el 50\% (Comision Europea, 2014). Aunque los jóvenes de la mayoría de los países europeos han visto aumentar su cualificación y formación, también se han visto envueltos en una mayor inestabilidad y precariedad laboral que, según diversos estudios, afecta de manera especialmente preocupante a países mediterráneos como Grecia, Italia y España (Benedicto, Echaves, Jurado, Ramos, \& Tejerina, 2016). La juventud española se ha visto obligada a asumir una nueva narrativa en la que es la principal damnificada de una crisis y un sistema sociopolítico incapaz de asegurar su integración en condiciones favorables (Benedicto et al., 2013). El elevado nivel de desempleo juvenil, la emigración forzada de sectores juveniles con alta formación, la incertidumbre y la frustración que produce un futuro incierto, son referencias permanentes en el discurso de los jóvenes españoles de los últimos años (Benedicto et al., 2016). Pero serán estos mismos jóvenes quienes, convertidos en jóvenes europeos legítimamente protagonistas de la tarea de construcción de la identidad europea, dibujen un futuro propio y al mismo tiempo global y cosmopolita para Europa.

En este contexto, el estudio que se presenta, en el marco del proyecto EU-2038: The Voice of the Youth, se plantea explorar la voz de la juventud española y observar y analizar su visión del futuro personal y de Europa. Se definen, para ello, dos objetivos: 1) Describir el análisis crítico que los y las jóvenes españolas hacen de Europa y su impacto en el desarrollo actual y futuro; y 2) Determinar el valor que otorgan a la educación como herramienta de promoción de valores y actitudes cívicas.

\section{Metodología \\ Participantes}

En este estudio participaron 268 jóvenes universitarios españoles $(64.6 \%$ chicas, $35.1 \%$ chicos, $0.4 \%$ otro) de entre 16 y 25 años (edad media= 20.72; DT = 2.71). La mayoría de ellos estaban recibiendo formación universitaria o formación profesional en el momento de la realización de la encuesta y cuentan con un nivel académico de Enseñanza Secundaria. La tabla 1 presenta las principales características definitorias de los y las participantes.

Tabla 1. Características sociodemográficas de los y las participantes

\begin{tabular}{|c|c|c|}
\hline & & Porcentaje/Media (D.T.) \\
\hline \multirow[t]{3}{*}{ Edad } & 16-18 años -preuniversitarios $(n=72)$ & $17.32(.71)$ \\
\hline & 19-22 años -universitarios o equivalente $(n=110)$ & $20.43(1.13)$ \\
\hline & $23-25$ años - postgrado o profesionales $(n=86)$ & $23.93(.81)$ \\
\hline \multirow[t]{3}{*}{ Sexo } & Chica $(\mathrm{n}=94)$ & $35.1 \%$ \\
\hline & Chico $(\mathrm{n}=173)$ & $64.6 \%$ \\
\hline & Otro $(n=1)$ & $0.4 \%$ \\
\hline \multirow[t]{2}{*}{ País de nacimiento } & España & $98.1 \%$ \\
\hline & Otro & $1.9 \%$ \\
\hline \multirow[t]{9}{*}{ Ocupación actual } & Estoy estudiando en el instituto/colegio & $21.3 \%$ \\
\hline & Estoy estudiando en la universidad/formación profesional (grado medio o superior) & $59 \%$ \\
\hline & Estoy trabajando a tiempo completo & $4.5 \%$ \\
\hline & Estoy trabajando a tiempo parcial & $10.1 \%$ \\
\hline & Estoy buscando trabajo & $12.3 \%$ \\
\hline & Estoy en un programa de prácticas & $2.2 \%$ \\
\hline & No estoy trabajando, haciendo prácticas ni estudiando & $0.4 \%$ \\
\hline & Estoy cuidando de niños o personas dependientes & $1.1 \%$ \\
\hline & Estoy esperando la resolución de mi solicitud de refugiado & $0 \%$ \\
\hline \multirow[t]{5}{*}{ Nivel académico } & No tengo ningún título académico & $0.4 \%$ \\
\hline & He obtenido el título de Educación Secundaria Obligatoria & $22.4 \%$ \\
\hline & He obtenido el título de Bachillerato & $45.5 \%$ \\
\hline & He obtenido un título de Ciclo Formativo (medio o superior) & $11.6 \%$ \\
\hline & Poseo un título universitario o superior & $20.1 \%$ \\
\hline
\end{tabular}




\section{Instrumentos}

Se utilizó una batería de instrumentos que ha sido validada en estudios previos con la muestra de referencia; con ella se evaluaban las siguientes variables:

- Información demográfica: preguntas abiertas relativas al género, edad, país de nacimiento, nacionalidad, país de residencia, país de nacimiento de la madre, país de nacimiento del padre, estado civil, ocupación actual y nivel académico.

- Prioridades con respecto al futuro (Strohmeier et al, 2017): se utilizó una escala compuesta por 19 ítems que pedía a los y las participantes que indicaran las cuestiones que consideraban más importantes, con un formato de respuesta de 5 puntos $(1=$ en absoluto; $5=$ mucho $)(\mathrm{a}=.78)$.

- Preocupaciones con respecto al futuro de Europa (Strohmeier et al, 2017): se utilizó una escala compuesta por 31 ítems que pedía a los y las participantes que indicaran las cuestiones que consideraban más importantes, con un formato de respuesta de 5 puntos ( 1 = en absoluto; $5=$ mucho) $(\mathrm{a}=.93)$.

- Visiones sobre el futuro de Europa (Strohmeier et al, 2017): se utilizó una escala compuesta por 39 ítems, que pedía a los y las participantes que indicaran las cuestiones que consideraban más importantes, con un formato de respuesta de 5 puntos ( 1 = nada importante; $5=$ muy importante) $(\mathrm{a}=.94)$.

- Valores (Ortega-Ruiz, Viejo, Gómez-López, en prensa): se utilizó un instrumento conformado por 12 ítems en el que los y las participantes expresaron su grado de acuerdo con diversas afirmaciones mediante un formato de respuesta de 5 puntos ( 1 = muy en desacuerdo; 5 = muy de acuerdo). Las dimensiones evaluadas fueron la Justicia, los Derechos Humanos, la Democracia y la Diversidad, tomadas como una puntuación global $(\mathrm{a}=.80)$.

- Actitudes (Ortega-Ruiz et al., en prensa): se utilizó un instrumento conformado por 18 ítems en el que los y las participantes expresaron su grado de acuerdo mediante un formato de respuesta de 5 puntos $(1=$ muy en desacuerdo; 5 = muy de acuerdo). Las dimensiones evaluadas fueron: (1) percepción de la eficacia $(\mathrm{a}=.66)$, (adaptación de Barrett y Zani, 2015); (2) apertura a la diversidad (adaptación de Alkheshnam, 2012) ( $\mathrm{a}=.89)$; (3) apertura a otras personas (adaptación de Bernard y Cronan, 1999) $(\mathrm{a}=.60) ;(4)$ intolerancia a la frustración (adaptación de Harrington, 2005) $(\mathrm{a}=.69)$.

\section{Procedimiento}

La recogida de datos se llevó a cabo mediante una encuesta online, disponible en la página web del proyecto (http://www. europe2038.eu/es/). Se requería en torno a 20-25 minutos para su cumplimentación. La recogida de datos se llevó a cabo entre los meses de junio y diciembre de 2016. Los participantes fueron reclutados a través de diversos eventos de difusión realizados en universidades, escuelas e institutos de la provincia de Córdoba y Sevilla, así como mediante la comunicación con organizaciones e instituciones políticas y sociales de todo el país en contacto directo con la juventud.

\section{Análisis de datos}

Los análisis descriptivos y comparativos fueron realizados mediante el software SPPS v.22. Para realizar la descripción de la muestra por edad, sexo y otras características sociodemográficas, así como para examinar el nivel de los y las jóvenes con relación a las diferentes dimensiones evaluadas, se utilizaron análisis descriptivos (porcentajes, medias y desviación típica). Posteriormente, mediante pruebas de comparación de medias para muestras independientes (prueba T), se exploraron las posibles diferencias significativas con relación a las prioridades y preocupaciones sobre el futuro, las visiones con respecto al futuro de Europa y las actitudes y valores mostrados por los y las participantes.

\section{Resultados}

\section{Análisis de Europa e impacto en el desarrollo actual y futuro}

Para abordar el primero de los objetivos, se solicitó a los y las participantes que analizaran su situación actual en el marco del contexto europeo y establecieran un ranking de los elementos que consideraban como sus prioridades para el futuro (Imagínate a ti mismo en el año 2038. ¿Cuáles de las siguientes cuestiones son más importantes para ti?. Por favor, marca también las que consideres tus cinco prioridades) y sus principales preocupaciones (Imagínate a ti mismo en el año 2038. ¿Cuáles de las siguientes cuestiones te preocupan?. Por favor, marca también las cinco que sean tus prioridades).

Los resultados obtenidos se presentan en la figura 1. El ranking de prioridades para el futuro ha señalado que el $62.8 \%$ de los participantes sitúan como prioridad principal ser feliz, seguido de estar sano $(60.5 \%)$, tener trabajo $(60.5 \%)$, tener una buena educación (28.6\%) y tener una relación sentimental feliz $(26.7 \%)$. Este top-5 varía ligeramente en cuanto a su orden si se atiende de forma independiente a las prioridades de chicas y chicos: el orden establecido por ellas sitúa a la salud $(66.5 \%)$ en un primer puesto, seguida de la felicidad (63.6\%) y el trabajo (62.4\%). El orden establecido por ellos sitúa a la felicidad (60.9\%) en el primer puesto, seguida del trabajo (56.5\%) y la salud (48.9\%). Ambos coinciden en otorgarle a la educación el quinto puesto en este orden de prioridades, si bien para ellas sentirse seguras en su país (30.6\%) ocupa el cuarto puesto del ranking, mientras que ellos se lo otorgan a tener una relación sentimental feliz (35.9\%). Diferencias estadísticamente significativas fueron halladas con respecto a la salud (t $(263)=-2.81 ; \mathrm{p}<.05)$, cuestión significativamente más importante para las chicas que para los chicos, y a tener una relación sentimental feliz $(\mathrm{t}(263)=2.57 ; \mathrm{p}<.05)$, siendo los chicos los que le otorgaron mayor importancia.

Con respecto a las principales preocupaciones de cara a su futuro, el $37.1 \%$ de los y las participantes señalaron que su principal preocupación era el terrorismo, mientras que el $35.5 \%$ se decantó por el desempleo. La pobreza se situaba en tercer puesto de este ranking (26.6\%), seguida de los prejuicios, la discriminación y el racismo $(24.4 \%)$ y la corrupción (20.6\%). También en estas preocupaciones existían diferencias entre ellas y ellos: en los primeros puestos de la clasificación de los chicos aparecían el terrorismo (32.6\%), el desempleo (29.2\%), el cambio climático $(23.6 \%)$, la corrupción $(22.5 \%)$ y el aumento de partidos de extrema derecha $(21.3 \%)$, mientras que en el ranking establecido por las chicas los cinco primeros puestos los ocupaban el terrorismo (39.9\%), el desempleo (39.2\%), la pobreza (29.7\%), los prejuicios, la discriminación y el racismo (27.2\%) y la violencia sexual $(22.8 \%)$. Con respecto a las diferencias significativas encontradas, la violencia sexual $(\mathrm{T}(245)=-2.25$; $\mathrm{p}>.005)$ preocupaba más a las chicas que a los chicos $(\mathrm{t}(245)=$ $-2.25 ; \mathrm{p}<.05)$, mientras que el aumento de partidos de extrema derecha, resultó ser más preocupante para los chicos $(\mathrm{t}(245)=$ $2.99 ; \mathrm{p}<.05)$. 
Figura 1. Prioridades y preocupaciones sobre el futuro: chicos y chicas

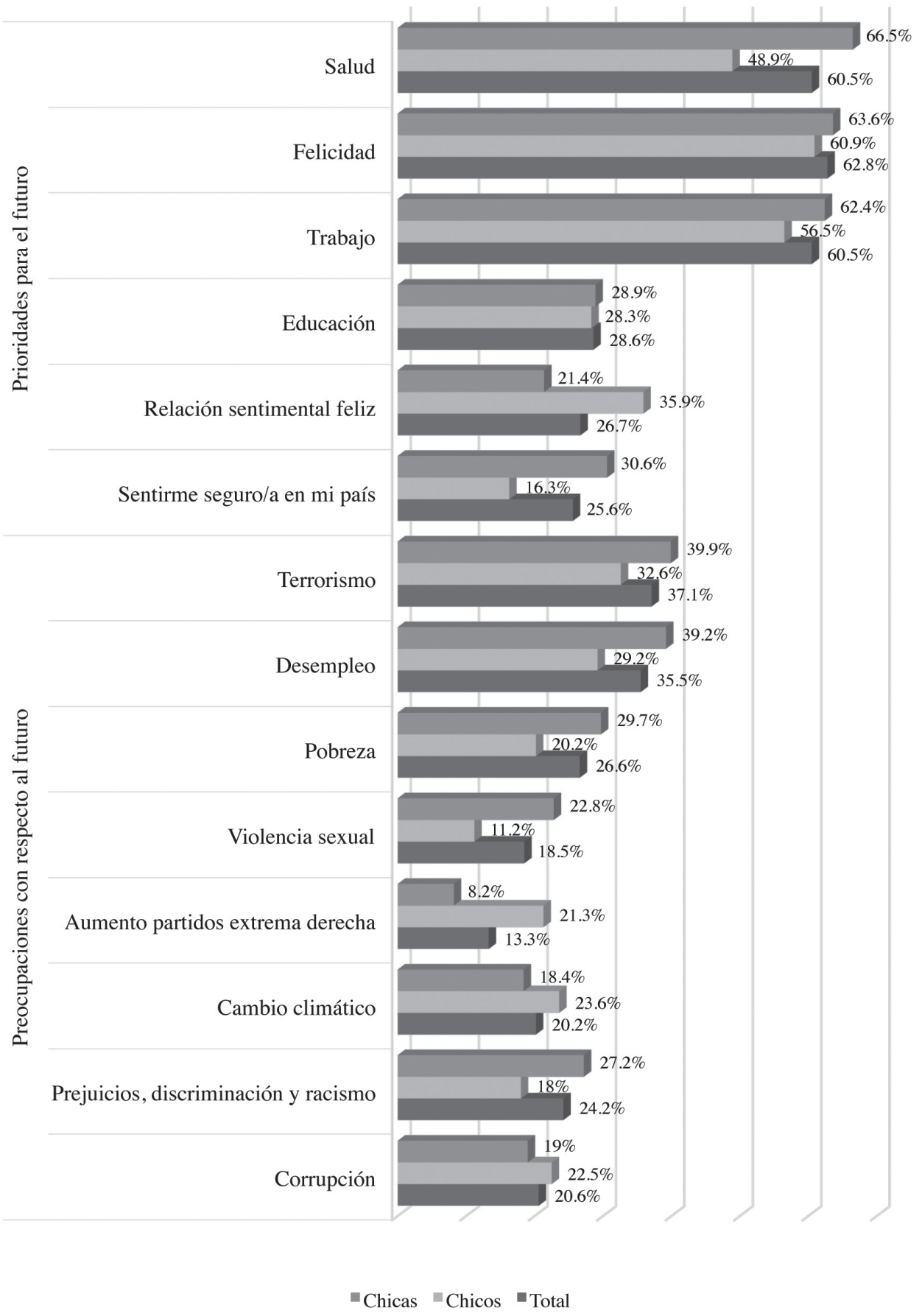

La educación como herramienta de promoción de valores y actitudes cívicas

El segundo de los objetivos se abordó con el análisis de variables como la aceptación de la diversidad cultural y promoción de la diversidad cultural, la asunción de la necesidad del progresivo cumplimiento de los Derechos Humanos y la profunda convicción de la democracia como régimen general sociopolítico, desde la perspectiva de que estos elementos están en la base de la cristalización de un comportamiento cívico ajustado (ver Figura 2).

Los resultados relativos a los valores aportaron datos referentes a cuatro ámbitos de principios y creencias interdependientes:
(1) la percepción de la justicia como un factor esencial en el logro de una sociedad igualitaria, (2) la consideración de los derechos humanos como un elemento fundamental a preservar y proteger, (3) el entendimiento del sistema democrático como la base de una sociedad equilibrada e íntegra y (4) la valoración de la diversidad cultural, de pensamiento, étnica, religiosa, etc. como un elemento positivo y beneficioso para la sociedad. Tratados de forma global, los y las jóvenes españoles mostraron un acuerdo global con esta dimensión de 4.64, lo que indica una muy alta identificación y orientación hacia los valores de justicia, democracia, derechos humanos y diversidad cultural, existiendo una diferencia significativa a favor de ellas, que puntuaban más alto $(\mathrm{t}(217)=-2.57, p<.05)$. 
En cuanto a las actitudes, se consideraron (1) el sentimiento de eficacia, formado a su vez por la eficacia interna percibida con respecto a su capacidad para comprender y analizar cuestiones relacionadas con Europa, la eficacia colectiva, o capacidad percibida para influir de manera conjunta en el futuro de Europa y la eficacia externa, entendida como la capacidad de Europa para hacer frente a las necesidades de los ciudadanos en general, y jóvenes en particular; (2) la apertura a la diversidad y hacia otras culturas, razas, etnias o procedencias; (3) la apertura a otras personas con diferentes formas de pensar y actuar; y (4) la intolerancia a la frustración ante la consecución de objetivos a largo plazo o difíciles de lograr. Consideradas estas actitudes de forma global, los y las participantes mostraron un acuerdo de 3.58, lo que supone un valor medio-alto, caracterizado por una marcada orientación hacia actitudes positivas, no existiendo diferencias significativas entre chicas y chicos.

Figura 2. Valores y actitudes cívicas de los chicos y chicas

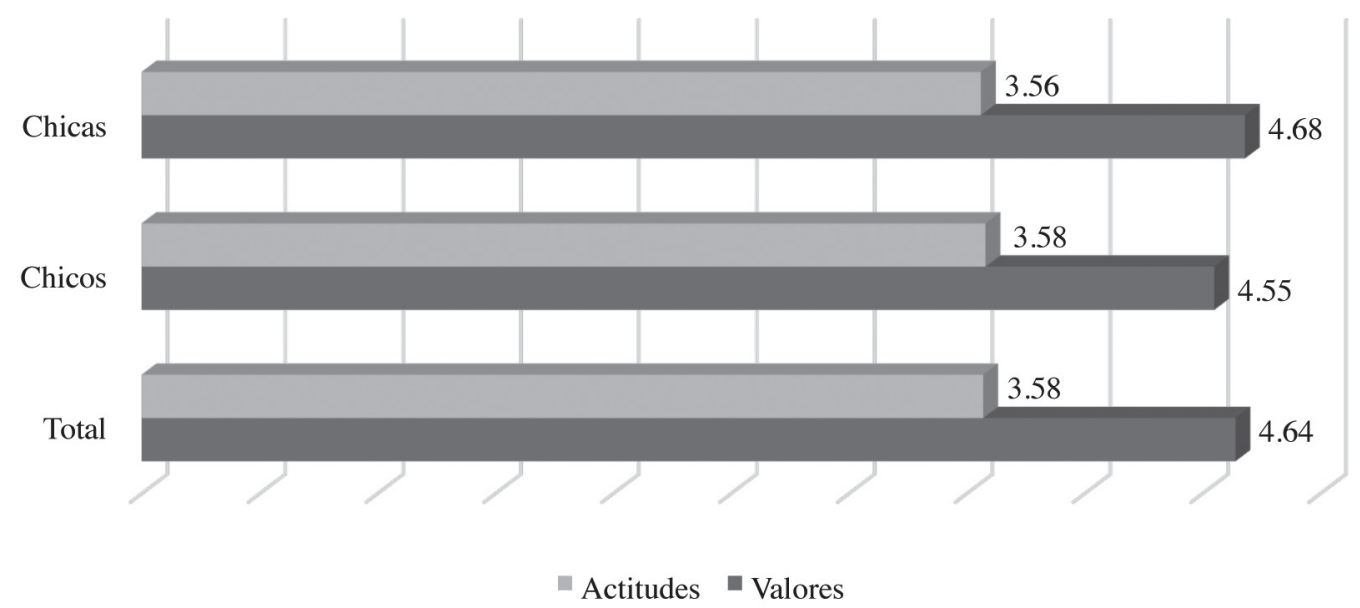

Finalmente, se abordó el papel de la educación en este entramado analizando las mejores estrategias de intervención y las principales áreas de actuación que los y las participantes identificaban, es decir, las líneas sobre las que pensaban que las políticas europeas debían trabajar para la construcción de un contexto cívico, comprometido, común, y equitativo en oportunidades, derechos y libertades.
Al igual que en los enunciados relativos a sus intereses y preocupaciones, se pidió a los y las participantes que ordenaran los ítems presentados por prioridad (Imagina que eres el líder de la Unión Europea y puedes establecer las prioridades de Europa en el año 2038. ¿Qué cuestiones son las más importantes?. Por favor, marca también las cuestiones que serían uno de sus tops 5), indicando las cinco cuestiones que consideraban más importantes (ver Figura 3).

Figura 3. Prioridades o herramientas de cambio para Europa

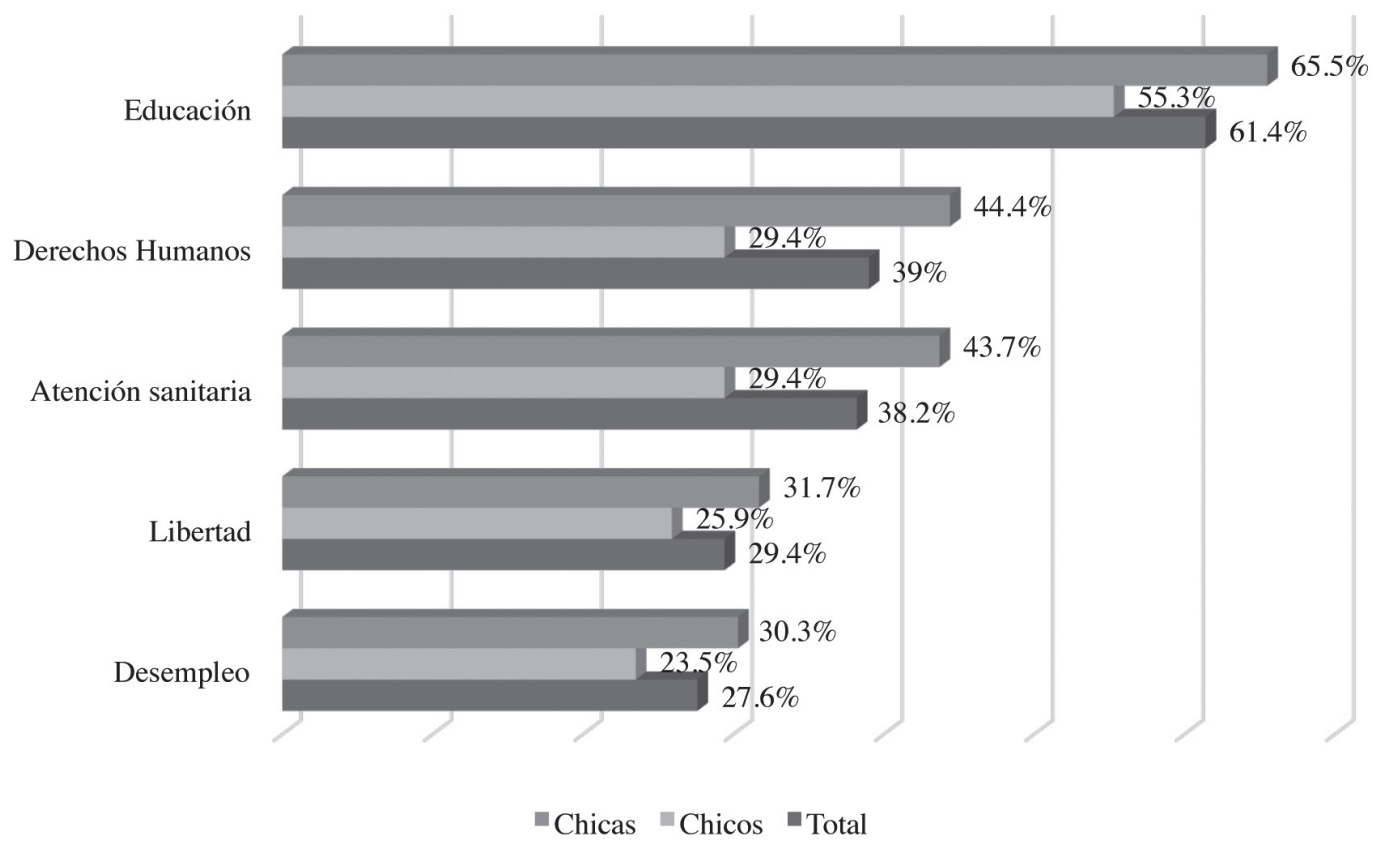


Según la clasificación general de la juventud participante, las cuestiones que actúan como herramientas de cambio y se deben promocionar son la educación (61.4\%), los Derechos Humanos (39\%), la atención sanitaria (38.2\%), la libertad (29.4\%) y el (des) empleo $(27.6 \%)$. Chicos y chicas mostraron un alto grado de acuerdo en este sentido, siendo que ambos posicionaban la educación en un primer lugar, seguida de los Derechos Humanos, la atención sanitaria, y la libertad; en el último puesto, ellos señalaban el medio ambiente y ellas señalaban, de nuevo, el (des) empleo. Diferencias significativas fueron halladas en relación con los Derechos Humanos (t $(225)=-2.25 ;<.05)$ y a la atención sanitaria $(\mathrm{t}(225)=-2.15 ;<.05)$, siendo las chicas las que le otorgaron mayor importancia.

\section{Conclusiones y discusión}

La universidad, como institución universalista, científica y docente tiene la obligación de incluir entre sus finalidades la formación de mentalidades críticas, constructivas y positivas que hagan de los jóvenes de hoy adultos futuros que sepan apostar por un mundo mejor. Un mundo mejor es un mundo organizado en torno a valores sociales de equidad y justicia, y la universidad tiene la obligación de incluir entre sus objetivos el dotar a los futuros y futuras ciudadanas de la competencia para saber elegir dichos valores. La globalización económica no ha sido capaz de incluir la mundialización de valores humanos de respeto profundo por la vida de las personas, su dignidad y su derecho a dar a la comunidad y recibir beneficios que sean buenos para todos. Los jóvenes son el eje central en la construcción de un futuro sólido y compartido por todos y la responsabilidad de Europa para con la población juvenil se torna evidente bajo esta consideración: la puesta en marcha de políticas y prácticas en pro de la creación de oportunidades y recursos se convierte en una tarea esencial. Es indispensable, por tanto, conocer qué análisis hacen los y las jóvenes respecto a la Europa actual, considerándolo de forma operativa a través del cuáles son las expectativas y prioridades que establecen con respecto a su presente y a su futuro, así como sus principales preocupaciones. Por ello, el primer objetivo de este estudio consistió en describir el análisis que los y las jóvenes hacían sobre Europa y sobre su impacto en el desarrollo actual y futuro. De forma general, los resultados de este estudio han confirmado las voces que han venido apuntando a la salud, la familia y la amistad como cuestiones de gran calado en las expectativas juveniles (CIS, 2002, 2008; INJUVE, 2016). Igualmente, distintos trabajos habían señalado ya la problemática laboral de las y los jóvenes españoles, cuestión de la que también se hizo eco la clasificación general realizada por los participantes: si bien el desempleo sigue siendo identificado como una de las preocupaciones más señaladas por los y las jóvenes participantes en relación a su futuro, -con los problemas económicos, las cuestiones relacionadas con el mundo laboral y, en particular, el paro entre los principales elementos a considerar (Benedicto et al., 2016; CIS, 2016; GETS, 2009)-, también la consideración de terrorismo o violencia generalizada en Europa en los últimos tiempos está causando un gran calado entre la juventud.

Más allá de la identificación de esta problemática, se hacía necesario avanzar en el conocimiento de los valores y actitudes cívicas que estos chicos y chicas demuestran, así como en el establecimiento de las que consideran como las mejores estrategias de intervención y las principales áreas de actuación que debe enfrentar la Unión Europea para continuar contribuyendo a la mejora y avance para la sociedad del siglo XXI.

Desde la compleja y completa teorización de Fishbein y Ajzen (1980) el conocimiento, actitudes y subjetividad constituyen elementos claves de las creencias, y estas, a su vez, son responsables en gran medida de la conducta social. La construcción de una identidad común y comprometida con un contexto social globalizado está profundamente relacionada con los valores y las actitudes, así como con la evidencia de los propios comportamientos. En el caso de los jóvenes, es particularmente importante considerar el impacto del contexto social inmediato y mediato, las personas con las que conviven y la cultura que ha ido conformando sus sistemas de creencias, pero también las experiencias sociales en las que han participado. Los chicos y chicas viven hoy en un mundo cada vez más interconectado y multicultural en el que las fronteras se desdibujan, siendo su aceptación o rechazo ante esta nueva realidad lo que podría determinar, en parte, el desarrollo de la Europa del futuro como una entidad de carácter supranacional y global. Un aumento en los niveles de identificación con Europa puede traducirse en una mayor implicación de la población juvenil en los procesos democráticos, políticos y cívicos que tienen lugar a su alrededor, tanto a nivel nacional como supranacional. En este sentido, cabría interpretar que las y los jóvenes españoles, caracterizados por una destacada orientación positiva y abierta hacia personas de otras culturas, razas, etnias o procedencias, consideran que una juventud unida puede conseguir influir en el desarrollo tanto de su futuro como del futuro de Europa, y manifiestan menos implicación personal ante necesidades individuales o frustraciones personales.

Por su parte, el segundo objetivo pretendía examinar la valoración de la educación por parte de los y las participantes como una herramienta de promoción de actitudes y valores cívicos. Los resultados destacaron la elevada puesta en valor que chicos y chicas hacen de la educación como principal herramienta de cambio y como prioridad de la Europa del futuro. Esto podría estar relacionado con resultados hallados en estudios previos, según los cuales existe un alto grado de acuerdo entre los jóvenes respecto a que las personas con mayor nivel académico tienen acceso a mejores oportunidades generales, y laborales en particular (INJUVE, 2016). Los jóvenes con estudios universitarios valoran más la formación académica como medio de prosperar, son más optimistas respecto a su propio futuro y están dispuestos a esperar para conseguir un trabajo acorde a su formación (INIU$\underline{\mathrm{VE}, 2016)}$. Esta elevada valoración de la educación por parte de la juventud eleva la responsabilidad de la universidad como institución que debe dar respuesta a necesidades tanto educativas, como sociales y competenciales. Una formación universitaria de calidad ha de procurar una coordinación efectiva entre la institución y los agentes implicados, entre los que se encuentra el estudiante (Ferra, López y Muñoz, 2018). De esta manera, tanto en niveles universitarios como preuniversitarios, es necesaria la adopción de estrategias innovadoras que faciliten el tránsito, la adaptación y la consolidación de chicos y chicas a un sistema cualitativamente diferente al de etapas educativas previas (Rodríguez-Muñiz y Díaz, 2015). A este respecto, estudios previos señalan que la coordinación entre la universidad y las instituciones educativas de educación secundaria es fundamental, así como la formación y sensibilización del profesorado en este ámbito (Lorenzo Moledo, Argos González, Hernández García y Vera Vila, 2014; Rodríguez-Muñiz y Díaz, 2015). La necesidad de que la universidad de convierta en un espacio seguro y beneficioso para los y las jóvenes se ha puesto de manifiesto en otras investigaciones, en las que se encontró que el alumnado experimentaba, entre otras, dificultades de comunicación con el profesorado, de comprensión de la terminología utilizada por los y las docentes, de conocimiento de la naturaleza de las materias o de desarraigo afectivo (García-Valcárcel y Tejedor, 2001; Ferra, López y Muñoz, 2018). Por tanto, asegurar de manera holística 
un tránsito exitoso por la etapa universitaria podría tener efectos positivos no sólo en la adquisición de conocimientos, sino también de valores y actitudes en jóvenes a los que la sociedad exigirá ser adultos competentes el día de mañana. Es por ello que la educación se erige como el pilar fundamental para lograr una cohesión social real, un exitoso mercado global y una verdadera cultura democrática europea, considerando que cumple un papel esencial para el bienestar futuro de los ciudadanos y las naciones que integran Europa (Comision Europea, 2014).Por último, este estudio representa un avance pionero en dar voz a las y los jóvenes españoles que serán los protagonistas de su propio futuro; esta población nos proporciona un perfil esperanzador: sus valores, su sensibilidad hacia la importancia de principios igualitarios, de apoyo mutuo y de solidaridad merecen ser registrados como fortalezas. No hay duda de que también esta investigación revela algunas debilidades que habrá que registrar y hacer de ellas una lectura comprensiva y correctiva: nuestros jóvenes son inseguros y se sienten vulnerables en cuanto a su competencia para abordar con entusiasmo su propio futuro profesional, y la investigación debería ahondar en esta línea. Estudios descriptivos de estos tópicos son necesarios no solo en el plano nacional, sino también como punto de partida para el diseño de trabajos cross-culturales que permitan evaluar el impacto de diferentes variables socioculturales. La visión del mundo que plantean estos chicos y chicas no es lo entusiasta y positiva que sus valores deberían hacerles ver. Las universidades españolas tienen que incluir entre sus objetivos una profunda internacionalización, para que la experiencia exterior, realizada con confianza y seguridad en sus propias competencias vaya dotando a nuestros jóvenes universitarios de esa seguridad en su proyecto vital que todavía, hoy, es una debilidad.

\section{Referencias}

Alkheshnam, A. (2012). Intercultural Competence: Components and Measurement (Unpublished PhD thesis). University of Surrey, UK.

Barrett, M., y Zani, B. (2015). Political and Civic Engagement. Multidisciplinary Perspectives. London, UK: Routledge.

Benedicto, J., Echaves, A., Jurado, T., Ramos, M., y Tejerina, B. (2016). Informe Juventud en España 2016. Madrid: INJUVE. Recuperado de http://www.injuve.es/sites/default/files/2017/24/ publicaciones/informe-juventud-2016.pdf

Benedicto, J., Mosteyrin de, L. F., Sastre, M. G., Pérez, A. M., Coppola, E. M., y Morán, M. L. (2013). Transitar a la intemperie: jóvenes en busca de integración. Madrid: INJUVE Recuperado de http://www.injuve.es/sites/default/files/Transitar_a_la_ intemperie.pdf

Bernard, E. M. y Cronan, F. (1999). The Child and Adolescent Scale of Irrationality: Validation Data and Mental Health Correlates. Journal of Cognitive Psychotherapy. 13(2), 121-132.

Brand-Gruwel, S., y Stadtler, M. (2011). Solving information-based problems: Evaluating sources and information. Learning and Instruction, 21, 175-179.

Britto, P. (2004). Análisis sociológico de la juventud española actual. Docencia e investigación: revista de la Escuela Universitaria de Magisterio de Toledo, 7-20. Recuperado de https://ruidera. uclm.es/xmlui/handle/10578/7931.

Centro de Investigaciones Sociológicas (CIS) (2002). Sondeo sobre la juventud española 2002 (primera oleada) (informe No. 2449). Recuperado de http://www.cis.es/cis/opencm/ES/1_encuestas/estudios/ver.jsp?estudio=2541

Centro de Investigaciones Sociológicas (CIS) (2008). Barómetro de junio de 2008 (Informe No. 2766). Recuperado de http://
www.cis.es/cis/opencm/ES/1_encuestas/estudios/ver.jsp?estudio $=8440$

Centro de Investigaciones Sociológicas (CIS) (2016). Barómetro de junio 2016. Recuperado de http://www.cis.es/cis/opencm/ ES/1_encuestas/estudios/ver.jsp?estudio=14289

Cicchelli, V. (2011). Les politiques de promotion de mobilités juvéniles en Europe. Informations Sociales, 3, 38-45.

Comision Europea (2014). Educacion, formacion, juventud y deporte. Recuperado de https://europa.eu/european-union/file/1071/ download_es?token=IkVzSy1E

Ferra, M. P., López, R. Q., y Galiano, I. M. (2018). Transición de Secundaria a la Universidad en estudiantes de los títulos de maestro de Educación Infantil y Primaria de la Universidad de Jaén. Aula Abierta, 47(2), 167-176. doi: https:// doi.org/10.17811/rifie.47.2.2018.167-176. Recuperado de https://www.unioviedo.es/reunido/index.php/AA/article/ view/12721/11685 Fishbein, M.y Ajzen, I. (1980). Understanding Attitudes and Predicting Social Behaviour. Englewood Cliffs, NJ: Prentice-Hall.

García-Valcárcel, A., y Tejedor, J. (2011). Variables TIC vinculadas a la generación de nuevos escenarios de aprendizaje en la enseñanza universitaria. Aportes de las curvas ROC para el análisis de diferencias. Educación XX1, 14(2), 43-78. doi: http://dx.doi.org/10.5944/educxx1.14.2.237.

Harrington, N. (2005). The Frustration Discomfort Scale: Development and psychometric properties. Clinical Psychology and Psychotherapy, 12, 374-387.

Huber, G. L. (2008). Aprendizaje activo y metodologías educativas. Revista de Educación, 59-81.

Instituto de la Juventud. Ministerio de Trabajo y Asuntos Sociales (2016). Informe INJUVE 2016. Recuperado de http://www. injuve.es/sites/default/files/2017/24/publicaciones/informe-juventud-2016.pdf

Jover, G. (2001). Educación y ciudadanía: el compromiso cívico de los jóvenes españoles. Revista electrónica Teoría de la Educación.

Kaluf, C. (2005). Diversidad Cultural: Materiales para la Formación Docente y el Trabajo de Aula. Chile: OREALC/UNESCO.

Kennedy, K. (1997). Citizenship education in review: Past perspectives and future needs. En K. Kennedy (Ed.), Citizenship Education and the Modern State. London: The Falmer Press.

Larrain, J. (2003). El concepto de identidad. Revista FAMECOS, 30-42.

López-Sidro, A. (2011). Reflexiones sobre los desafíos de Bolonia y la misión del profesor universitario. Entre la innovación y la tradición. Revista Jurídica de Investigación e Innovación Educativa, 4, 23-44. Recuperado de https://dialnet.unirioja.es/servlet/articulo? codigo $=4584931$.

Lorenzo Moledo, M., Argos González, J., Hernández García, J. y Vera Vila, J. (2014). El acceso y la entrada del estudiante a la universidad: Situación y propuestas de mejora facilitadoras del tránsito. Educación XX1, 17(1), 15-38. Recuperado de http://revistas.uned.es/index.php/educacionXX1/article/ view/9951.

Marín, M.A. (2003). La dimensión europea de la educación. En I. M. Gómez-Chacón (Ed.). Identidad Europea. Individuo, Grupo, Sociedad (pp. 81-96). Bilbao: Universidad de Deusto.

Ministerio de Asuntos Exteriores y de Cooperación (MAEC) (2018). España y la Unión Europea. Recuperado de http://www. exteriores.gob.es/Portal/es/PoliticaExteriorCooperacion/ UnionEuropea/Paginas/EspUE.aspx

Moreno, A. (2015). Produciendo la juventud: la imagen de los jóvenes en los estudios generales sobre la juventud española. Revista Estudios de Juventud, 35-48. 
Ortega-Ruiz, R., Viejo, C., y Gómez-López, M. (en prensa). Europe 2038: The Voice of Spanish Youth. In D. Strohmeier and H. Tenenbaum. Young people's visions and worries for the future of Europe. Findings from the Europe 2038 project. UK: Routledge

Palmer, A., Montaño, J.J., y Palou, M. (2009). Las competencias genéricas en la educación superior. Estudio comparativo entre la opinión de empleadores y académicos. Psicothema, 21, 433-438.

Rodríguez-Izquierdo, R. M. (2014). Modelo formativo en el Espacio Europeo de Educación Superior: valoraciones de los estudiantes. Aula Abierta, 42(2), 106-113. doi: https:// doi.org/10.17811/rifie.42.2014.106-113. Recuperado de https://www.unioviedo.es/reunido/index.php/AA/article/ view/11658
Rodríguez-Muñiz, L. J., y Díaz, P. (2015). Estrategias de las universidades españolas para mejorar el rendimiento en matemáticas del alumnado de nuevo ingreso. Aula abierta, 43(2), 69-76. doi: https://doi.org/10.17811/rifie.43.02.2015.69-76. Recuperado de https://www.unioviedo.es/reunido/index.php/ AA/article/view/11681

Santiago, P., Tremblay, K., Basri, E., y Arnal, E. (2008). Tertiary education for the knowledge society (Vol. 2). Paris: OECD.

Strohmeier, D., Barrett, M., Bora, C., Caravita, S., Donghi, E., Dragoti, E., ... y Viejo, C. (2017). Young people's engagement with the European Union: The importance of visions and worries for the future of Europe. Zeitschrift fur Psychologie, 225, 313-323. doi: https://doi.org/10.1027/2151-2604/a000314.

Tobón, S., Rial, A., Carretero, M.A. y García, J.A. (2006). Competencias, Calidad y Educación Superior. Bogotá: Cooperativa Editorial Magisterio. 
